\title{
Canine Gastrointestinal Nematodiases and Associated Risk Factors in Kigali City, Rwanda
}

\author{
Pie Ntampaka $\mathbb{D}^{1},{ }^{1}$ François Niragire $\mathbb{D}^{D},{ }^{2}$ Philip Njeru Nyaga, ${ }^{3}$ and Gervais Habarugira $\mathbb{D}^{1}$ \\ ${ }^{1}$ Department of Veterinary Medicine, University of Rwanda, P.O. Box 57, Nyagatare, Rwanda \\ ${ }^{2}$ Department of Applied Statistics, University of Rwanda, P.O. Box 1514, Kigali, Rwanda \\ ${ }^{3}$ Department of Veterinary Pathology, Microbiology and Parasitology, University of Nairobi, P.O. Box 29053-00625, Nairobi, Kenya
}

Correspondence should be addressed to Pie Ntampaka; piusynt@gmail.com

Received 24 March 2021; Revised 7 June 2021; Accepted 2 July 2021; Published 26 July 2021

Academic Editor: José F. Silveira

Copyright (c) 2021 Pie Ntampaka et al. This is an open access article distributed under the Creative Commons Attribution License, which permits unrestricted use, distribution, and reproduction in any medium, provided the original work is properly cited.

Canine nematodes pose a public health risk to humans and livestock; however, the prevalence of canine nematodiases in Rwanda is unknown. This study aimed at determining the prevalence of canine nematodiases and identifying the risk factors for such infections in Kigali, the capital city of Rwanda. A cross-sectional study involved 93 dogs selected across Kigali city. Faecal samples were collected from apparently healthy dogs, and nematode eggs were identified and quantified using the McMaster technique. Risk factors for canine nematodiases were analysed by a multivariable binary logistic regression model. The overall prevalence of gastrointestinal (GI) nematodiases in dogs was 33.3\% (95\% CI: 23.8-42.9). The most prevalent species was Ancylostoma spp with $32.3 \%$ (95\% CI: 22.8-41.8). Nearly $38.7 \%$ and $3.2 \%$ of the dogs infected with Ancylostoma spp and Toxocara canis had high egg counts per gram (EPG) of faeces $(\geq 550)$, respectively. Approximately $96.8 \%$ of dogs infected with nematodes had monoinfection. Logistic regression analysis showed that dog's age (1 to 2.5 years old), location (Gasabo and Kicukiro districts), and feeding practices were significantly associated with prevalence of canine nematodiases. In particular, the adjusted odds ratio (AOR) was more than 5 times higher for dogs fed on uncooked animal products and leftovers from households and restaurants compared to those who ate food prepared for them. The AOR was also about 16 times higher for dogs that scavenged and ate leftovers from households compared to those who ate food prepared for them. The findings of this study indicate that the prevalence of GI nematodes in domestic dogs in Kigali city, Rwanda, was 33.3\% (95\% CI: 23.8-42.9). The identified nematodes, namely, Ancylostoma spp. and Toxocara canis, are zoonotic, and dogs and humans are at risk of contracting these nematodes. The factors associated with canine GI nematodes in Kigali city include feeding practices and the dog's age and location (district). Dog owners need to rethink procedures for deworming and feeding their dogs. Again, the public should be made aware of the role of dogs in transmitting zoonotic nematodes to humans.

\section{Introduction}

Dogs play a considerable role in helping humans to improve quality of life [1]. It has been demonstrated that pet dog owners are healthier than nonowners [2,3]. Pet dogs can help people under stressful conditions in enjoying their recreation and curing some pathological conditions such as high blood pressure [4]. Also, people can own dogs for various reasons such as business, hunting, herding livestock, and guarding. Dogs also offer a variety of services such as helping the disabled live independently and search and rescue missions as well as sniffing drugs and explosive detection
[5]. Although dogs have become an indispensable companion, they also constitute a potential source of a variety of human infections [1].

Dogs can harbour parasitic infections, thus transmitted between livestock and humans including helminths and protozoa [6,7]. Ascarids and Ancylostomatidae have been frequently reported to be the main helminths of dogs and cats with global significance [8]. Dogs contract ancylostomiasis through skin penetration or oral ingestion of infective larvae. Oral infection occurs through ingestion of infected milk or paratenic hosts or while being suckled [9]. Canine toxocariasis can be transmitted via ingestion of faecal material or soil 
contaminated with viable embryonated eggs, transplacentally, or ingestion of milk from infected dams while being suckled [10].

Humans can contract nematodes through ingesting items contaminated with embryonated eggs or infective larvae. Also, infective larvae can be transmitted through the cutaneous route as well as mosquito bites (e.g., Filariae). Ancylostoma spp that affects dogs can cause cutaneous larva migrans while Toxocara canis can cause visceral larva migrans and ocular larva migrans in humans $[11,12]$. Studies conducted around the world reported the prevalence of canine GI nematodiases that varied between $9.5 \%$ and $51 \%[13,14]$.

A wide range of factors can influence the prevalence of canine helminthiases including intrinsic factors such as age, sex, and breed or extrinsic ones, for instance, feeding, environment, accuracy of testing, regular deworming, and geographical location [15-19]. The control of canine nematodiases and helminthiases at large consists of proper hygiene, regular preventive deworming, and treatment of clinically ill individuals $[20,21]$. However, misuse of anthelminthics may lead to the emergence of resistance to the drugs used for the treatment of animal and human helminthiases [20]. Anthelminthic resistance can primarily be prevented through applying evidence-based treatment, respecting the dosage and adherence to proper management strategies [8].

A study conducted in Egypt found that alkaline $\mathrm{pH}$ of soil influenced the occurrence of soil-transmitted helminths (e.g., Toxocara spp and Ancylostomatidae). Soil properties, for instance, temperature, moisture, $\mathrm{pH}$, and organic matter, may influence the prevalence of soil-transmitted helminths [22]. For example, the development of eggs of Ancylostomatidae requires a temperature varying between 20 and $30^{\circ} \mathrm{C}$ and suitable shade and moisture alongside clay-sandy soil $[22,23]$. In Rwanda, soils are naturally fragile and derived from schistose, quartzite, gneissic, granite, and volcanic rocks. Again, the country's $88 \%$ of the soil $\mathrm{pH}$ is acidic (pH3.5-6.5) [24, 25]. Despite the potential importance of canine nematode infections as a one health concern, there are no reports on their infections in Rwanda. Thus, this study aimed at determining the prevalence of GI nematodes in dogs and associated risk factors in Kigali city, Rwanda.

\section{Materials and Methods}

2.1. Study Sites. This study was conducted in Kigali city, Rwanda, from September 2016 to March 2017. In Rwanda, the climate is tropical, and the country has four climatic zones, including the central plateau, where Kigali city is situated [24]. The plateau has an annual rainfall and a temperature ranging from 1,100 to $1,300 \mathrm{~mm}$ and 18 to $20^{\circ} \mathrm{C}$, respectively [24]. One report showed that the relative humidity in Kigali ranges between $61 \%$ and $85 \%$ in August and April, respectively [26]. In the suburb of Kigali, soils are hill ferro and valley histosoil types. Much of the soil across Kigali is also acidic [25]. Administratively, Kigali city is subdivided into three districts, and each district is in turn subdivided into administrative sectors [27]. The present study covered nine sectors that were selected from the three districts of Kigali city; each district was represented by three sectors.
Figure 1 shows the map of Kigali city with district and sector level boundaries.

2.2. Study Design and Sample Size. This cross-sectional study involved collecting data on management practices and faecal samples from dogs.

We determined the sample size based on the national dog population of 18,117 reported in 2016 [28]. The number of dogs in Kigali represented 2,157, thus $11.9 \%$ of the national dog population [29]. Due to a lack of previous studies on canine helminthiases in Rwanda, the prevalence of canine nematodiases was assumed to be $50 \%$.

Based on previous population-based health studies such as the Rwanda demographic and health survey, where the response rate has generally remained above 95\% [30], we expected a relatively high response rate. Thus, we increased the sample size by only $10 \%$ to cater for possible nonresponse $[31,32]$. The minimal sample size $(n)$ of dogs needed for testing hypothesis on risk factors for nematodiases in this study was thus estimated using Cochran's formula for determining sample size for proportions [33], as follows:

$$
\begin{aligned}
\frac{Z^{2} p(1-p) / e^{2}}{1+Z^{2} \cdot p(1-p) / e^{2} N} & =\frac{1.96^{2} \times 0.50^{2} / 0.10^{2}}{1+1.96^{2} \cdot 0.50^{2} / 0.10^{2} \times 2157} \\
& =\frac{96.04}{1+96.04 / 2157}=91.94 \cong 92 \mathrm{dogs},
\end{aligned}
$$

where $N$ is the population size and $e$ is the level of precision. After adjusting for a $10 \%$ nonresponse rate (9 additional dogs), the present study targeted a total sample of 101 dogs. Based on district-level registers for the dog population, which showed households owning dogs, we obtained a listing of all dogs for each of the selected sectors. We selected this study sample through a two-stage sampling procedure. In the first stage, we considered the dog population per administrative sector and selected three administrative sectors with the largest dog population from each of the three districts of Kigali city. Thus, we chose nine sectors across Kigali city, namely, Gatenga, Niboye, and Kicukiro in the Kicukiro district; Kacyiru, Kimironko, and Gisozi in Gasabo district; and Mageragere, Nyamirambo, and Kigali in Nyarugenge district. In the second stage, systematic random sampling helped choose the households at the sector level. Cooperation with local authorities allowed locating the target households. Given some households owned many dogs, we randomly considered one dog per household for data collection.

2.3. Data Collection. The study was approved by the Rwanda National Ethics Committee (Ethical approval 115/RNEC/ 2017). Dog owners were explained about this study and signed written consent before participating.

Data were successfully collected from 93 dogs, and a questionnaire was used to collect data on dogs (age, sex, breeds, and location) and on dog keeping practices (frequency of deworming, feeding practices, and control of dog movements) [34]. Faecal samples were collected directly from the rectum using a gloved finger and kept in faecal jars. 


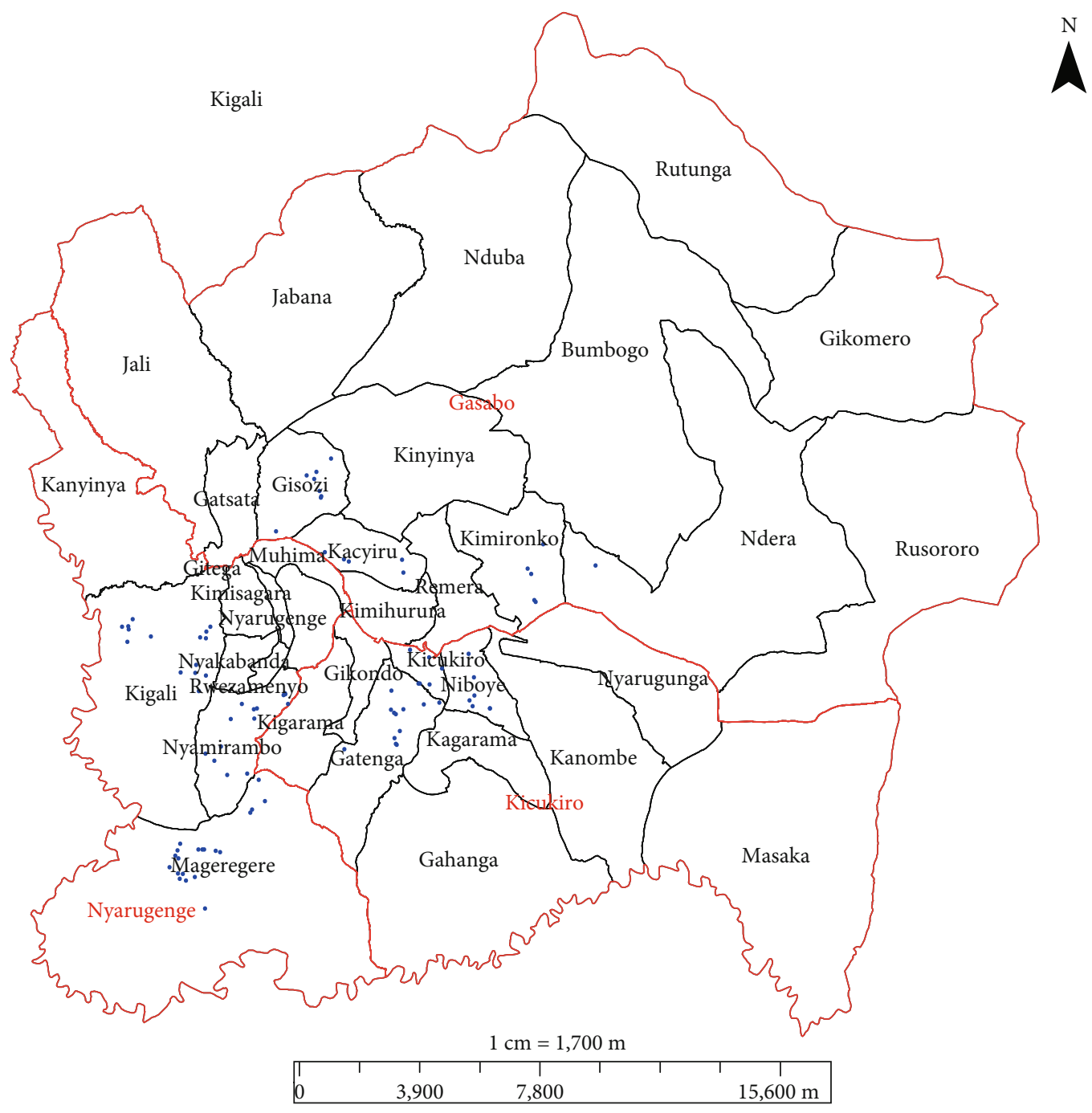

Legend

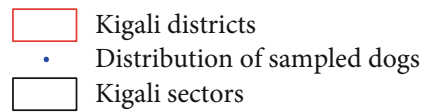

Figure 1: Administrative districts (red boundaries) and sectors (black boundaries) of Kigali city. The blue dots show the location of households owning sampled dogs across the study sites. The locations are Kigali, Nyamirambo, and Mageragere sectors of Nyarugenge district; Kicukiro, Niboye, and Gatenga sectors of Kicukiro district; and Gisozi, Kimironko, and Kacyiru sectors of Gasabo district. Data on the location of each study dog was collected using GPS and allowed generating the map using ArcGis10.2 software.

All samples were stored in a cool box and were analysed at the laboratories of the Rwanda Agriculture and Animal Resources Development Board (RAB).

2.4. Faecal Analysis. The preparation of float fluid involved dissolving sodium chloride (Park Scientific Limited, UK) in tap water. The flotation fluid had a specific gravity of 1.200 ; that is, $400 \mathrm{~g}$ of sodium chloride was dissolved in a litre of water. The analysis was done on the day of sampling using the McMaster technique as previously described by Hansen and Perry [35]. Nematode eggs were identified by examining the sample under a light microscope at 10x magnification based on shape, thickness of shell, and presence of morulae [36].

2.5. Data Processing and Analysis. Data were entered and then analysed in the IBM SPSS Statistics for Windows, version 20. EPG of faeces was obtained by multiplying the number of eggs by a factor of 50 as previously described by Hansen and Perry [35]. The infection was quantified by EPG which was grouped into light infection (50-100 EPG), moderate infection (150-500 EPG), and heavy infection ( $\geq 550$ EPG) [37]. The analysis of faecal samples resulted in a binary response variable that indicated whether a sampled 
TABLE 1: Prevalence of nematodes in analysed faecal samples ( $n=93$ dogs).

\begin{tabular}{|c|c|c|c|c|}
\hline \multirow{2}{*}{ Nematode species } & \multirow{2}{*}{ Number of infected dogs } & \multirow{2}{*}{ Percent (\%) } & \multicolumn{2}{|c|}{ 95\% CI for percentage } \\
\hline & & & Lower limit & Upper limit \\
\hline Ancylostoma spp & 30 & 32.3 & 22.757 & 41.759 \\
\hline Toxocara canis+Ancylostoma spp & 1 & 1.1 & 0.0 & 3.171 \\
\hline Total & 31 & 33.3 & 23.752 & 42.914 \\
\hline
\end{tabular}

dog was infected or not infected. These data were used to determine infection prevalence. To investigate associations between selected factors and prevalence of canine nematodiases, data were analysed using a multivariable binary logistic regression model as described previously [38, 39]. The backward variable selection procedure was applied to selected variables for the best fitting model for the data at hand [40]. The 5\% level of significance was used to interpret the results regarding the association. The 95\% confidence intervals for the AORs were used to assess the significance and direction of the associations.

\section{Results}

3.1. Characteristics of Study Dogs. Faecal samples were collected from 93 apparently healthy dogs from different locations and of different ages, sex, and breeds.

Nearly $15.1 \%$ and $29 \%$ were $<1$ year old and 1 to 2.5 years old, respectively, while $31.2 \%$ and $24.7 \%$ aged $>2.5$ to 5 years (a dog infected with ascarids belonged to this category) and $>5$ years, respectively. Some study dogs received regular or irregular deworming. Some dogs were scavengers or fed on food prepared for them or leftovers from households or restaurants. Also, some dogs were restricted while others were not restricted. The occurrence of gastrointestinal nematodes in study dogs is indicated (Table 1).

The prevalence of nematodiases in dogs was 33.3\% (95\% CI: 23.8-42.9). The predominant species was Ancylostoma spp with $32.3 \%$ (95\% CI: 22.8-41.8) (Table 1). Of all the 31 infected dogs, $96.8 \%$ were parasitised with one species of nematodes while one dog was infected with two species of nematodes. Parasitic load in dogs suffering from ancylostomiasis and toxocariasis in Kigali city, Rwanda, is shown (Figure 2).

Chi-square tests of the associations of the occurrence of canine nematodiases with the selected potential risk factors in Kigali city and corresponding $p$ values are presented in Table 2.

All the variables in Table 2 were considered for the backward variable selection procedure that was based on a multivariable logistic regression model [40]. The results showed that only three factors, namely, feeding practices, dog's age and location, were significantly related to the infection. The results in Table 3 show the AORs and the corresponding 95\% confidence intervals.

The results in Table 3 show that the prevalence of canine nematodiases in Kigali city was significantly associated with the dog's age ( 1 to 2.5 years old), location, and feeding practices. The AOR of nematodiases was more than 10 times higher for dogs that were aged 1-2.5 years $(A O R=10.732$;

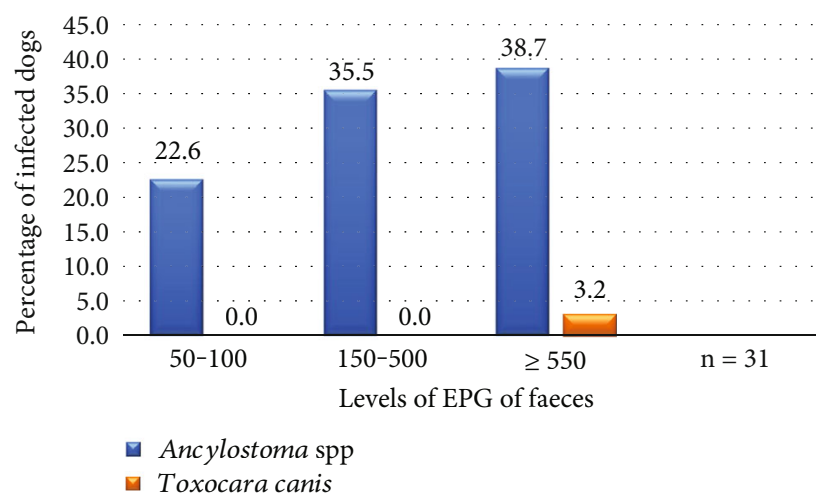

FIgURe 2: Parasitic egg load in dogs suffering from ancylostomiasis and toxocariasis in Kigali city. Counts $\geq 500$ EPG were found in $38.7 \%$ of dogs infected with Ancylostoma spp. The one dog infected with $T$. canis was also infected with hookworm $(1 / 31)$ and had count $\geq 500$ EPG. In addition, $35.5 \%$ and $22.6 \%$ of those infected with Ancylostoma spp had moderate egg load and light egg load, respectively (Figure 2).

95\% CI: 1.510-76.263) compared to those who were younger than one year old.

Compared to dogs located in Nyarugenge district, the AORs of the infection were about 22 times higher in dogs located in Gasabo district (AOR $=21.617$; 95\% CI: 4.242$110.160)$ and about 12 times higher in dogs located in Kicukiro district $(\mathrm{AOR}=11.959 ; 95 \% \mathrm{CI}$ : 2.534-56.445). Besides, the AOR was more than 5 times higher for dogs fed on uncooked animal products and leftovers from households and restaurants compared to those who ate food prepared for them.

The odds of canine nematodiases were also about 16 times higher for dogs that scavenged and ate leftovers from households compared to those who ate food prepared for them.

\section{Discussion}

The prevalence of canine nematodiases in Kigali city was 33.3\% (95\% CI: 23.8-42.9). The dog's age, location (district), and feeding practices were statistically significant risk factors associated with the canine nematodiases. Our findings can help policymakers to strategize effective control measures in dog populations and to inform dog owners of the role of dogs in transmitting zoonotic nematodiases to humans.

Our overall prevalence was lower but comparable to 51\% reported by Yacob et al. [14] in Ethiopia. However, it was higher than 9.5\% reported by Wright et al. [13] in England. 
TABLE 2: Chi-square tests of the associations of the occurrence of canine gastrointestinal nematodiases with the selected potential risk factors in Kigali city.

\begin{tabular}{|c|c|c|c|c|}
\hline \multirow{2}{*}{ Sample characteristics } & \multicolumn{3}{|c|}{ Canine nematodes } & \multirow{2}{*}{$p$ value } \\
\hline & Present $(\%)$ & Absent (\%) & Total (\%) & \\
\hline \multicolumn{5}{|l|}{ Deworming frequency } \\
\hline Less than twice a year & $21(22.6)$ & $38(40.9)$ & $59(63.4)$ & \multirow{3}{*}{0.824} \\
\hline At least twice a year & $4(4.3)$ & $9(9.7)$ & $13(14.0)$ & \\
\hline Irregularly & $6(6.5)$ & $15(16.1)$ & $21(22.6)$ & \\
\hline \multicolumn{5}{|l|}{ Feeding practices } \\
\hline Food prepared for dogs & $8(8.6)$ & $22(23.7)$ & $30(32.3)$ & \multirow{3}{*}{0.183} \\
\hline Uncooked animal products, leftovers from households and restaurants & $15(16.1)$ & $33(35.5)$ & $48(51.6)$ & \\
\hline Scavenging and leftovers from households & $8(8.6)$ & $7(7.5)$ & $15(16.1)$ & \\
\hline \multicolumn{5}{|l|}{ Control of movements } \\
\hline Nonrestricted & $\begin{array}{c}12(12.9) \\
1(1.1)\end{array}$ & $21(22.6)(19.4)$ & $33(35.5)$ & \multirow[t]{2}{*}{0.646} \\
\hline Restricted & $19(20.4)$ & $41(44.1)$ & $60(64.5)$ & \\
\hline \multicolumn{5}{|l|}{ Breed } \\
\hline Local & $9(11.8)$ & $19(20.4)$ & $28(30.1)$ & \multirow[t]{2}{*}{0.873} \\
\hline Pure or cross & $22(23.7)$ & $43(46.2)$ & $65(69.9)$ & \\
\hline \multicolumn{5}{|l|}{ Age group } \\
\hline$<1$ year & $2(2.2)$ & $12(12.9)$ & $14(15.1)$ & \multirow{4}{*}{0.138} \\
\hline $1-2.5$ years & $13(14)$ & $14(15.1)$ & $27(29)$ & \\
\hline$>2.5-5$ years & $10(10.8)$ & $19(20.4)$ & $29(31.2)$ & \\
\hline$>5$ years & $6(9.7)$ & $17(18.3)$ & $23(24.7)$ & \\
\hline \multicolumn{5}{|l|}{ Sex } \\
\hline Female & $7(7.5)$ & $17(18.3)$ & $24(25.8)$ & \multirow[t]{2}{*}{0.802} \\
\hline Male & $24(25.8)$ & $45(48.4)$ & $69(74.2)$ & \\
\hline \multicolumn{5}{|l|}{ Study district } \\
\hline Nyarugenge & $8(8.6)$ & $39(41.9)$ & $47(50.5)$ & \multirow{3}{*}{0.003} \\
\hline Gasabo & $10(10.8)$ & $9(9.7)$ & $19(20.5)$ & \\
\hline Kicukiro & $14(15.1)$ & $13(14)$ & $14(29)$ & \\
\hline
\end{tabular}

TABLE 3: Factors influencing canine nematodiases in Kigali city.

\begin{tabular}{|c|c|c|c|c|}
\hline \multirow{2}{*}{ Variable } & \multirow{2}{*}{ Categories } & \multirow{2}{*}{ Adjusted odds ratio (AOR) } & \multicolumn{2}{|c|}{ 95\% C.I. for AOR } \\
\hline & & & Lower & Upper \\
\hline \multirow{4}{*}{ Dog's feeding practices } & Food prepared for dogs & 1 & \multicolumn{2}{|c|}{ Reference } \\
\hline & $\begin{array}{l}\text { Uncooked animal products, leftovers from } \\
\text { households and restaurants }\end{array}$ & 5.354 & 1.205 & 23.776 \\
\hline & Scavenging and leftovers from households & 15.646 & 2.349 & 104.191 \\
\hline & $<1$ year old & 1 & \multicolumn{2}{|c|}{ Reference } \\
\hline \multirow{2}{*}{ Dog's age } & 1-2.5 years old & 10.732 & 1.510 & 76.263 \\
\hline & $>2.5-5$ years old & 3.923 & 0.597 & 25.779 \\
\hline \multirow{5}{*}{ Location } & $>5$ years old & 4.765 & 0.643 & 35.301 \\
\hline & Nyarugenge & 1 & \multicolumn{2}{|c|}{ Reference } \\
\hline & Gasabo & 21.617 & 4.242 & 110.160 \\
\hline & Kicukiro & 11.959 & 2.534 & 56.445 \\
\hline & Constant & 0.006 & - & - \\
\hline
\end{tabular}

Management practices and environmental factors might have influenced the difference in prevalence in these various studies. Around $46.2 \%$ of this study dogs received regular or irregular deworming, while $83.9 \%$ ate food provided by the owners. However, none of the dogs investigated by Yacob et al. received anthelminthics. Although some of the dogs 
investigated by Wright et al. got anthelminthics, none received such drugs in the three weeks before faecal sampling. Given Ancylostoma spp and Toxocara canis are geohelminths, the acidic $\mathrm{pH}$ of the soil in Kigali may negatively impact their prevalence. One study found alkaline $\mathrm{pH}$ of the soil influences the occurrence of soil-transmitted helminths [22].

The prevalence of Ancylostoma spp was comparable to $34.8 \%$ found by Davoust et al. [41] in Gabon. However, it was higher than $24.6 \%$ reported by Ayinmode et al. [42] in Nigeria and lower than $93.8 \%$ reported by Schandevyl et al. [43] in the Democratic Republic of Congo (DRC).

The difference in prevalence may be related to management practices and accuracy in coprological testing. For instance, most dogs involved in the study by Schandevyl et al. [43] in the DRC were not properly looked after and they all were in poor condition. Furthermore, Schandevyl et al. [43] performed both McMaster technique and larval culturing to detect Ancylostoma spp.

Different species belonging to Ancylostomatidae can infect dogs including A. caninum, A. braziliense, A. ceylanicum, and Uncinaria stenocephala [44]. Of these, A. braziliense, A. caninum, and $U$. stenocephala can cause cutaneous larva migrans (sand-worm disease) in humans, while A. ceylanicum can cause eosinophilic enteritis. Similarly, A. caninum has been reported to cause eosinophilic enteritis, but it rarely matures into an adult in the human small intestine [12, 45].

Studies conducted in Rwanda reported human ancylostomiasis prevalence that varies between $6.33 \%$ and $33 \%$ $[46,47]$. Counts $\geq 500$ EPG were found in $38.7 \%$ of dogs infected with Ancylostoma spp. One dog infected with $T$. canis was also infected with hookworm and had count $\geq$ 500 EPG. These dogs could shed a high number of eggs in the environment and potentially put people at risk of contracting ancylostomiasis and toxocariasis.

Given the challenges to distinguish species of Ancylostoma based on egg morphometry and that a host can be parasitised by several species concurrently [44], the investigation of human ancylostomiasis in Rwanda should consider the risk of exposure to faeces deposited by walking and wandering dogs. The present study prevalence of Toxocara canis was lower than $9.8 \%$ reported by Ayinmode et al. [42] in Nigeria. Toxocara canis antibodies have been detected in people across Africa. For instance, two previous studies conducted in preschool children aged between 9 months and 5 years old in Nigeria and children aged 1-15 years old in Ghana detected Toxocara canis antibodies in $37.3 \%$ and $53.5 \%$ of the study children, respectively $[48,49]$. Further, one study conducted in various groups of professionals in Egypt detected anti-T. canis antibodies in $24 \%$ of the professionals [50].

Although there are no published data about human toxocariasis in Rwanda, the dog suffering from toxocariasis and ancylostomiasis in this study had an EPG of 750 for Toxocara canis. The high level of infection in the tested dog $(E P G \geq 550)$ suggests that the dog could shed a high number of eggs in the environment and potentially put people at risk of developing visceral larva migrans and ocular larva migrans [12]. Like a previous study by Yacob et al. [14] in Ethiopia, this study found that the dog's age correlated with the prevalence of nematodiases. The present study also found a corre- lation between the dog's location and the prevalence of canine nematodiases. Besides, our findings were consistent with those of Abere et al. [18] in Ethiopia, who reported that feeding practices positively correlated with the prevalence of canine helminthiases.

Single time point faecal sampling in this study may have impacted the prevalence of canine nematodiases. Thus, the data in this study is a snapshot prevalence which may not necessarily represent the true burden of canine nematodiases. A kinetic study could shed some light on the prevalence and the dynamics of nematodiases in dogs. We also used the egg detection method (McMaster technique), but it would not detect prepatent infections. Even centrifuging faecal samples before examining them can miss numerous hookworm infections [51]. Further studies using molecular laboratory techniques would be more suitable.

\section{Conclusions}

The findings of this study indicate that the prevalence of GI nematodes in domestic dogs in Kigali city, Rwanda, was 33.3\% (95\% CI: 23.8-42.9). The identified nematodes, namely, Ancylostoma spp. and Toxocara canis, are zoonotic, and dogs and humans are at risk of contracting these nematodes. The factors associated with canine GI nematodes in Kigali city include feeding practices and the dog's age and location (district). Dog owners need to rethink procedures for deworming and feeding their dogs. Again, the public should be made aware of the role of dogs in transmitting zoonotic nematodes to humans.

\section{Data Availability}

The data collected and analysed to support the findings of this study are included in the article. The questionnaire used during the data collection is available at https://doi.org/10.21203/rs.3.rs$26155 / \mathrm{v} 4$, and the source is cited at a relevant place within the text as a reference [34].

\section{Conflicts of Interest}

The authors declare that there is no conflict of interest regarding this paper.

\section{Acknowledgments}

The authors would like to thank the management of the districts of Kigali city for authorising data collection. We are also grateful to dog owners for frank collaboration during data collection. Furthermore, the authors acknowledge the leadership of the Rwanda Agriculture and Animal Resources Development Board for authorising use of the facilities of the National Veterinary Laboratory. Part of this work was funded by BHEARD, award number BFS-G-11-00002, and the predecessor fund the Food Security and Crisis Mitigation II grant, award number EEM-G-00-04-00013. The funder sponsored data collection, that is, buying reagents and materials and paying transport costs during the collection of faecal samples. 


\section{References}

[1] D. L. Wells, "Domestic dogs and human health: an overview," British Journal of Health Psychology, vol. 12, no. 1, pp. 145156, 2007.

[2] W. P. Anderson, C. M. Reid, and G. L. Jennings, "Pet ownership and risk factors for cardiovascular disease," The Medical Journal of Australia, vol. 157, no. 5, pp. 298-301, 1992.

[3] J. Serpell, "Beneficial effects of pet ownership on some aspects of human health and behaviour," Journal of the Royal Society of Medicine, vol. 84, no. 12, pp. 717-720, 1991.

[4] B. Headey and M. M. Grabka, "Pets and human health in Germany and Australia: National longitudinal results," Social Indicators Research, vol. 80, no. 2, pp. 297-311, 2007.

[5] A. I. Wandeler, C. N. Macpherson, and F. X. Meslin, Dogs, Zoonoses, and Public Health, CABI Pub, 2000.

[6] S. V. Soriano, N. B. Pierangeli, I. Roccia et al., "A wide diversity of zoonotic intestinal parasites infects urban and rural dogs in Neuquen, Patagonia, Argentina," Veterinary parasitology, vol. 167, no. 1, pp. 81-85, 2010.

[7] R. Sharma, B. Singh, J. Gill, E. Jenkins, and B. Singh, "Canine parasitic zoonoses in India: status and issues," Revue scientifique et technique (International Office of Epizootics), vol. 36, no. 3, pp. 817-830, 2017.

[8] D. Traversa, "Pet roundworms and hookworms: a continuing need for global worming," Parasites \& Vectors, vol. 5, no. 1, p. 91, 2012.

[9] L. R. Ballweber, Veterinary Parasitology (Practical Veterinarian), Butterworth-Heinemann Publication, 1st edition edition, 2001.

[10] D. Despommier, "Toxocariasis: clinical aspects, epidemiology, medical ecology, and molecular aspects," Clinical Microbiology Reviews, vol. 16, no. 2, pp. 265-272, 2003.

[11] L. S. Roberts and J. Janovy, Gerald D. Schmidt \& Larry S. Roberts' Foundations of Parasitology, McGraw-Hill, New York, USA, 8th Edition edition, 2009.

[12] D. D. Despommier, D. O. Griffin, R. W. Gwadz, P. J. Hotez, and C. A. Knirsch, Parasitic Diseases, Parasites Without Borders, Inc. NY, New York, 7th edition edition, 2019.

[13] I. Wright, K. Stafford, and G. Coles, "The prevalence of intestinal nematodes in cats and dogs from Lancashire, north-west England," The Journal of Small Animal Practice, vol. 57, no. 8, pp. 393-395, 2016.

[14] H. Yacob, T. Ayele, R. Fikru, and A. Basu, "Gastrointestinal nematodes in dogs from Debre Zeit, Ethiopia," Veterinary Parasitology, vol. 148, no. 2, pp. 144-148, 2007.

[15] T. Ilić, Z. Kulišić, N. Antić, K. Radisavljević, and S. Dimitrijević, "Prevalence of zoonotic intestinal helminths in pet dogs and cats in the Belgrade area," Journal of Applied Animal Research, vol. 45, no. 1, pp. 204-208, 2016.

[16] D. Barutzki and R. Schaper, "Results of parasitological examinations of faecal samples from cats and dogs in Germany between 2003 and 2010," Parasitology Research, vol. 109, no. S1, pp. 45-60, 2011.

[17] D. Neves, L. Lobo, P. B. Simões, and L. Cardoso, "Frequency of intestinal parasites in pet dogs from an urban area (Greater Oporto, northern Portugal)," Veterinary Parasitology, vol. 200, no. 3-4, pp. 295-298, 2014.

[18] T. Abere, B. Bogale, and A. Melaku, "Gastrointestinal helminth parasites of pet and stray dogs as a potential risk for human health in Bahir Dar town, north-western Ethiopia," Veterinary World, vol. 6, no. 7, p. 388, 2013.

[19] I. Robertson, P. Irwin, A. Lymbery, and R. Thompson, "The role of companion animals in the emergence of parasitic zoonoses," International Journal for Parasitology, vol. 30, no. 1213, pp. 1369-1377, 2000.

[20] T. Pullola, J. Vierimaa, S. Saari, A.-M. Virtala, S. Nikander, and A. Sukura, "Canine intestinal helminths in Finland: prevalence, risk factors and endoparasite control practices," Veterinary Parasitology, vol. 140, no. 3-4, pp. 321-326, 2006.

[21] W. Kollataj, A. Milczak, B. Kollataj, I. D. Karwat, M. Sygit, and K. Sygit, "Risk factors for the spread of parasitic zoonoses among dog owners and their families in rural areas," Annals of Agricultural and Environmental Medicine, vol. 19, no. 1, pp. 79-84, 2012.

[22] S. E. Etewa, S. A. Abdel-Rahman, N. F. Abd El-Aal, G. M. Fathy, M. A. El-Shafey, and A. M. G. Ewis, "Geohelminths distribution as affected by soil properties, physicochemical factors, and climate in Sharkyia governorate Egypt," Journal of Parasitic Diseases, vol. 40, no. 2, pp. 496-504, 2016.

[23] M. Mabaso, C. Appleton, J. Hughes, and E. Gouws, "The effect of soil type and climate on hookworm (Necator americanus) distribution in KwaZulu-Natal, South Africa," Tropical Medicine \& International Health, vol. 8, no. 8, pp. 722-727, 2003.

[24] Republic of Rwanda, Rwanda Compendium of Environment Statistics, Rwanda Environment Management Authority, Kigali, 2019.

[25] N. Nabahungu, "Rwandan Soil Health Status for Sustainable Food Security and Economic Growth," 2016.

[26] Royal HaskoningDHV, Environmental and Social Impact Assessment of the Kigali Wastewater Project, Royal HaskoningDHV, Luxembourg, 2016.

[27] National Institute of Statistics of Rwanda, Statistical Yearbook 2014, Republic of Rwanda, 2014.

[28] The New Times, Three thousand dogs culled in Rwanda in 2016, The New Times, 2017.

[29] P. Ntampaka, P. N. Nyaga, F. Niragire, J. Gathumbi, and M. Tukei, "Knowledge, attitudes and practices regarding rabies and its control among dog owners in Kigali city, Rwanda," PloS One, vol. 14, no. 8, article e0210044, 2019.

[30] National Institute of Statistics of Rwanda, "Ministry of Health of Rwanda, and ICF International," in Rwanda Demographic and Health Survey 2014-15, NISR, MOH, and ICF International, Rockville, Maryland, USA, 2016.

[31] J. Kotrlik and C. Higgins, "Organizational research: determining appropriate sample size in survey research appropriate sample size in survey research," Information Technology, Learning, and Performance Journal, vol. 19, p. 43, 2001.

[32] A. A. Al-Subaihi, "Sample size determination influencing factors and calculation strategies for survey research," Saudi Medical Journal, vol. 24, no. 4, pp. 323-330, 2003.

[33] W. Cochran, "Double sampling," in Cochran WG Sampling Techniques, pp. 327-358, N Y John Wiley Sons Inc., 1977.

[34] P. Ntampaka, F. Niragire, P. N. Nyaga, and G. Habarugira, "Canine helminthiases and associated risk factors in Kigali city Rwanda," Preprint, 2020.

[35] J. Hansen and B. D. Perry, "The epidemiology, diagnosis, and control of helminth parasites of ruminants," A handbook, 1994.

[36] E. J. L. Soulsby, A Textbook of Helminths, Arthropods, and Protozoa of Domesticated Animals, ELBS with Bailliere Tindall, London, 7th edition, 1982. 
[37] R. I. Rodríguez-Vivas, E. Gutierrez-Ruiz, M. E. Bolio-González et al., "An epidemiological study of intestinal parasites of dogs from Yucatan, Mexico, and their risk to public health," Vectorborne and zoonotic diseases, vol. 11, no. 8, pp. 1141-1144, 2011.

[38] C. Y. J. Peng and T. S. H. So, "Logistic regression analysis and reporting: a primer," Understanding Statistics: Statistical Issues in Psychology, Education, and the Social Sciences, vol. 1, pp. 31-70, 2002.

[39] J. C. Stoltzfus, "Logistic regression: a brief primer," Academic Emergency Medicine, vol. 18, no. 10, pp. 1099-1104, 2011.

[40] M. Z. I. Chowdhury and T. C. Turin, "Variable selection strategies and its importance in clinical prediction modelling," Family medicine and community health, vol. 8, no. 1, article e000262, 2020.

[41] B. Davoust, T. Normand, O. Bourry, H. Dang, E. Leroy, and G. Bourdoiseau, "Epidemiological survey on gastro-intestinal and blood-borne helminths of dogs in north-east Gabon," The Onderstepoort Journal of Veterinary Research, vol. 75, no. 4, pp. 359-364, 2008.

[42] A. B. Ayinmode, O. O. Obebe, and E. Olayemi, "Prevalence of potentially zoonotic gastrointestinal parasites in canine faeces in Ibadan, Nigeria," Ghana medical journal, vol. 50, no. 4, pp. 201-206, 2016.

[43] P. Schandevyl, T. Mbundu, and W. Sumbu, "Prevalence of intestinal parasites in dogs in Kinshasa, Zaire," Annales de la Societé belge de Médecine tropicale, vol. 67, no. 4, pp. 369374, 1987.

[44] D. D. Bowman, S. P. Montgomery, A. M. Zajac, M. L. Eberhard, and K. R. Kazacos, "Hookworms of dogs and cats as agents of cutaneous larva migrans," Trends in Parasitology, vol. 26 , no. 4, pp. 162-167, 2010.

[45] A. P. Oliveira-Arbex, E. B. David, T. C. Oliveira-Sequeira, S. Katagiri, S. T. Coradi, and S. Guimarães, "Molecular identification of Ancylostoma species from dogs and an assessment of zoonotic risk in low-income households, São Paulo State, Brazil," Journal of helminthology, vol. 91, no. 1, pp. 14-19, 2017.

[46] E. Ivan, N. J. Crowther, E. Mutimura, L. O. Osuwat, S. Janssen, and M. P. Grobusch, "Helminthic infections rates and malaria in HIV-infected pregnant women on anti-retroviral therapy in Rwanda," PLoS Neglected Tropical Diseases, vol. 7, no. 8, article e2380, 2013.

[47] E. Ruberanziza, D. Mupfasoni, B. Karibushi et al., “A recent update of Schistomiasis mansoni endemicity around Lake Rweru," Rwanda Medical Journal, vol. 68, pp. 5-9, 2010.

[48] O. A. Sowemimo, Y.-L. Lee, S. O. Asaolu et al., "Seroepidemiological study and associated risk factors of Toxocara canis infection among preschool children in Osun State, Nigeria," Acta tropica, vol. 173, pp. 85-89, 2017.

[49] G. Kyei, I. Ayi, J. Boampong, and P. Turkson, "Sero-epidemiology of Toxocara canis infection in children attending four selected health facilities in the central region of Ghana," Ghana Medical Journal, vol. 49, no. 2, pp. 77-83, 2015.

[50] M. A. Awadallah and L. M. Salem, "Zoonotic enteric parasites transmitted from dogs in Egypt with special concern to Toxocara canis infection," Veterinary world, vol. 8, no. 8, pp. 946957, 2015.

[51] K. Stafford, T. M. Kollasch, K. T. Duncan et al., "Detection of gastrointestinal parasitism at recreational canine sites in the USA: the DOGPARCS study," Parasites \& Vectors, vol. 13, no. 1, article 4147, pp. 275-310, 2020. 